\title{
SIMULATION OF THE TWO-BODY PROBLEM IN GEOGEBRA
}

\author{
Danka LUČIĆ, Mario VARGA \\ Department of Mathematics and Informatics, Faculty of Sciences, University of Novi Sad, Trg Dositeja Obradovića 3, \\ 21000 Novi Sad, Serbia, e-mail: danka.lucic@ hotmail.com,mario90v@ hotmail.com
}

\begin{abstract}
Classical problem of the motion of two bodies under gravitational interaction will be analyzed and simulated in GeoGebra. The two-body problem will be reduced to the single-body problem in central force field. Solutions of the single-body problem will be mapped onto solutions of the two-body problem and their correspondence will be analyzed. Finally, simulation of the two-body problem in a moving frame will be shown.
\end{abstract}

Keywords: two-body problem, simulation, GeoGebra, central force

\section{INTRODUCTION}

The Kepler problem for the motion of the planet around the Sun is one of the oldest problems of classical mechanics. It sevres as a paradigmatic problem by which one can study the fundamental laws of physics and apply different mathematical tools. Recently, there were considerable efforts to use simple computer algebra systems to analyze the Kepler problem and enlighten its physical and mathematical aspects, alike. We will mention only those contributions which are related to the application of GeoGebra system. Murillo [1] studied the relation between elliptic trajectory of the planet and the force acting on it using GeoGebra. Kovács [2] demonstrated the numerical capabilities of GeoGebra by applying it to Kepler problem. Finally, Hašek [3] used the Feynman's solution of the planetary motion problem and used it to simulate the elliptic orbit around the Sun and demonstrate the angular momentum conservation.

In this paper we will observe two point particles that interact only with each other. In order to find paths of these two particles, we will reduce the two-body problem to the single-body problem with reduced mass. Then we will find trajectory of one particle, and how the trajectory of the other particle depend on it.

We will show that trajectories of the particles are conic sections, but also, how trajectories looks like when observed from another inertial frame of reference, i.e., when center of mass of the system is moving. The motion will be simulated in GeoGebra.

\section{THE TWO BODY PROBLEM}

\subsection{Motivation}

In classical mechanics, the two-body problem is to determine the motion of two point particles that interact only with each other. Common examples include the Moon orbiting the Earth, Earth orbiting the Sun, but also, a classical electron orbiting an atomic nucleus. Interesting is the fact that the stars move around each other, and that $85 \%$ of them in the galaxy is in binary systems or triplets, different from the Sun which is an isolated star. In this work we will present a model of this binary systems.

\subsection{Mathematical Solution}

The solution described below is given in [4]. We will observe two particles $A$ and $B$. These two particles attract each other by a Newton's gravitational force which is directly proportional to the masses and inversely proportional to the square of the distance between them (Fig 1). Here:

- $m_{a}, m_{b}$ are masses of particles $A$ and $B$;

- $\vec{r}_{a}, \vec{r}_{b}$ are radius vectors of particles' $A$ and $B$ positions;

- $M=m_{a}+m_{b}$ is the total mass of the system;

- $\vec{R}$ is the radius vector of the center of mass;

$M \vec{R}=m_{a} \vec{r}_{a}+m_{b} \vec{r}_{a}$.

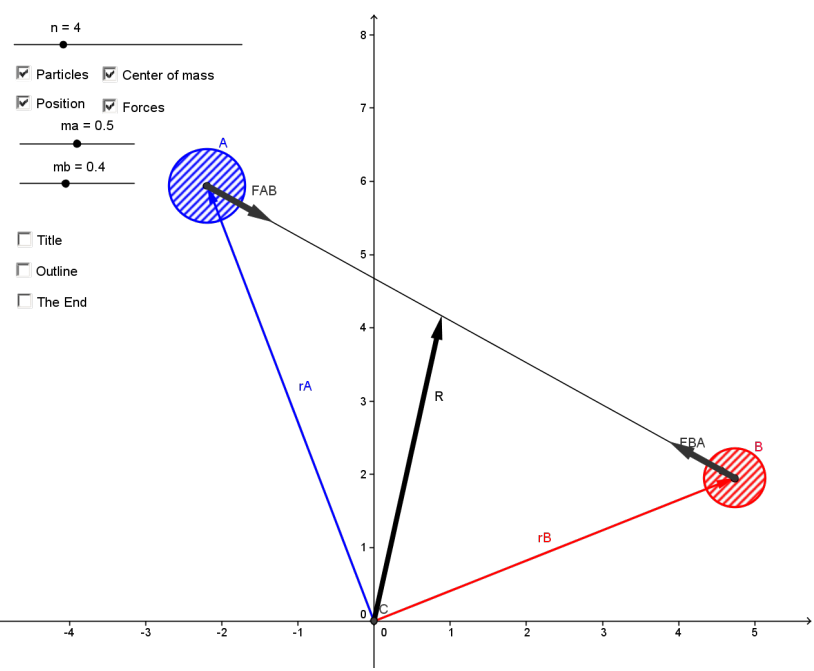

Fig. 1 Particles in the inertial frame

Newton's gravitational force is

$$
\vec{F}_{A B}=G \frac{m_{a} m_{b}}{r^{2}} \vec{e}, \quad \vec{F}_{B A}=-\vec{F}_{A B} .
$$

There are no external forces, therefore it can be shown that the center of mass moves uniformly. As a result of this, we can choose the origin of our inertial frame to be exactly the center of mass. With this we get a much less complicated form of our problem. If we put the origin at the center of mass, in the equation (1), where the center of 
mass was defined the left-hand side will be $\overrightarrow{0}$, so we can easily express $\vec{r}_{a}$ as a function of $\vec{r}_{b}$ :

$\vec{r}_{a}=-\frac{m_{b}}{m_{a}} \vec{r}_{b}$

This is the key for our solution, because we will find the trajectory of $B$ and, using relation (2), we will map it onto the trajectory of $A$.

Using the previous result and subtracting it with Newtons second law

The motion of the particles is determined by Newton's law:

$m_{a} \ddot{\vec{r}}_{a}=\vec{F}_{A B}, \quad m_{b} \ddot{\vec{r}}_{b}=\vec{F}_{B A}$.

The distance $r$, which appears in the gravitational forces $\vec{F}_{A B}$ and $\vec{F}_{B A}$, must be expressed in terms of radius vectors of particles, $\vec{r}_{a}=-r_{a} \vec{e}, \vec{r}_{b}=r_{b} \vec{e}$, and reads $r=r_{b}-r_{a}$. Taking into account these relations and equation 22, one may subtract the equations of motion (3) and after some straightforward calculations get the following differential equation:

$$
\mu \ddot{\vec{r}}_{b}=-G \frac{\mu m}{r_{b}^{2}} \vec{e}
$$

where $\mu$ (reduced mass) and $m$ are:

$$
\mu=\frac{m_{b} m_{a}}{m_{a}+m_{b}}, \quad m=\frac{m_{a}^{3}}{\left(m_{a}+m_{b}\right)^{2}} .
$$

The two body problem is reduced to a single body problem, which is usually solved in polar coordinates $r$ and $\theta$. We could try to find $r$ and $\theta$ as functions of time, but it is much easier to find directly $r$ as the function of $\theta$. If we introduce a new function

$$
u(t)=\frac{1}{r(t)}
$$

where $t$ is time, the previous equation can be rewritten as the following Binet differential equation:

$$
\frac{\mathrm{d}^{2} u}{\mathrm{~d} \theta^{2}}+u=\frac{G m}{h^{2}}
$$

where $h=r^{2} \dot{\theta}=$ const .

Solving this differential equation, we easily get the trajectory of particle $B$ :

$$
r_{b}(\theta)=\frac{\frac{G m}{r_{b 0}^{2} v_{0}^{2}}}{1+\left(\frac{r_{b 0} v_{0}^{2}}{G m}-1\right) \cos \theta} .
$$

Using the relation (2) we get the trajectory of particle $A$ :

$r_{a}(\theta)=\frac{\frac{-m_{b} G m}{m_{a} r_{b 0}^{2} v_{0}^{2}}}{1+\left(\frac{r_{b 0} v_{0}^{2}}{G m}-1\right) \cos \theta}$.

\subsection{Simulation in GeoGebra}

One can notice that the shape of our trajectory depends on masses of both of the particles and also on initial values of velocity and position of particle $B$. The shape of the trajectory depends on the value

$$
e=\frac{r_{b 0} v_{0}^{2}}{G m}-1
$$

called eccentricity.

If we change initial data, we change $e$ and thereby we change trajectories of particle $B$ and $A$. If we choose initial data so that $e$ has some of the following values, we get:

$$
\begin{array}{cl}
e=0 & \text { circle; } \\
0<e<1 & \text { ellipse; } \\
e=1 & \text { parabola; } \\
e>1 & \text { hyperbola. }
\end{array}
$$

In order to show trajectories in GeoGebra, we use sliders to change initial data, and also masses of our particles. Coordinates of particles $A$ and $B$ are given by

$$
B\left(r_{b}(\theta), \theta\right), \quad A\left(r_{a}(\theta), \theta+\pi\right) .
$$

In the following examples different kinds of trajectories are shown:

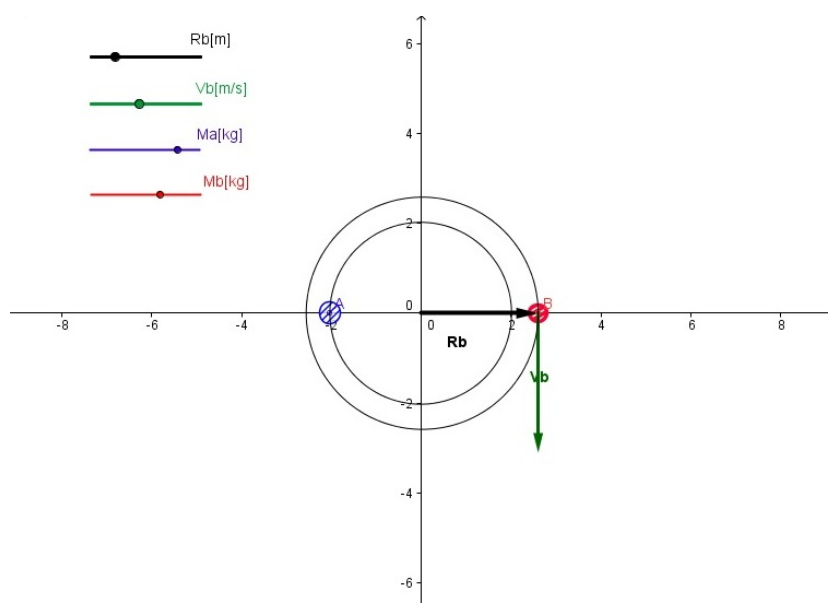

Fig. 2 Trajectory - circle

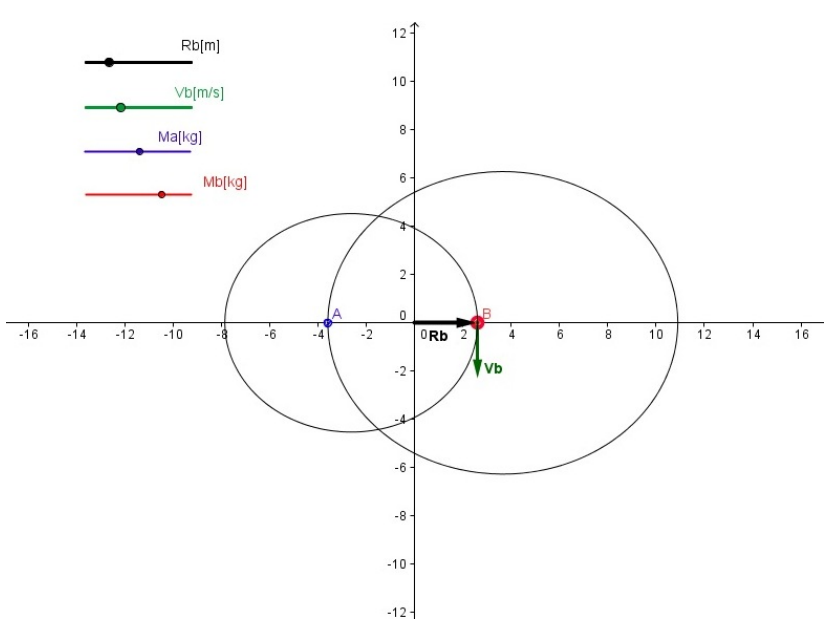

Fig. 3 Trajectory - ellipse 


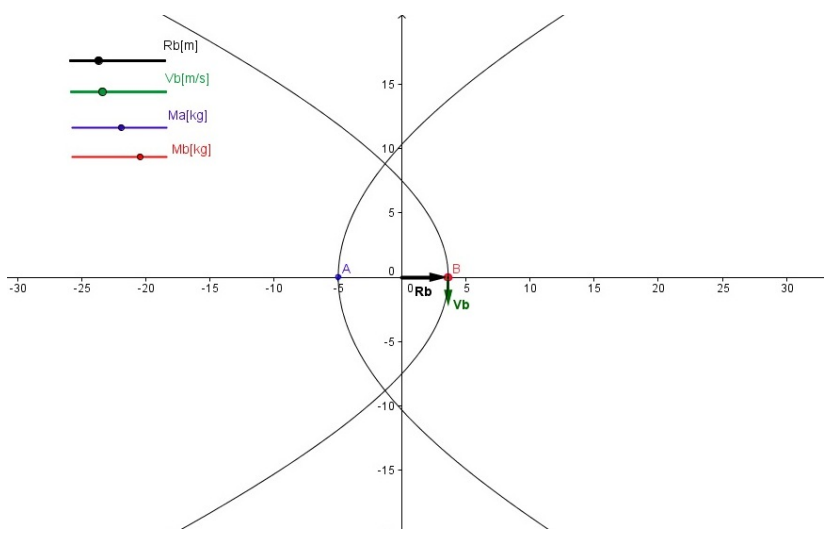

Fig. 4 Trajectory - parabola

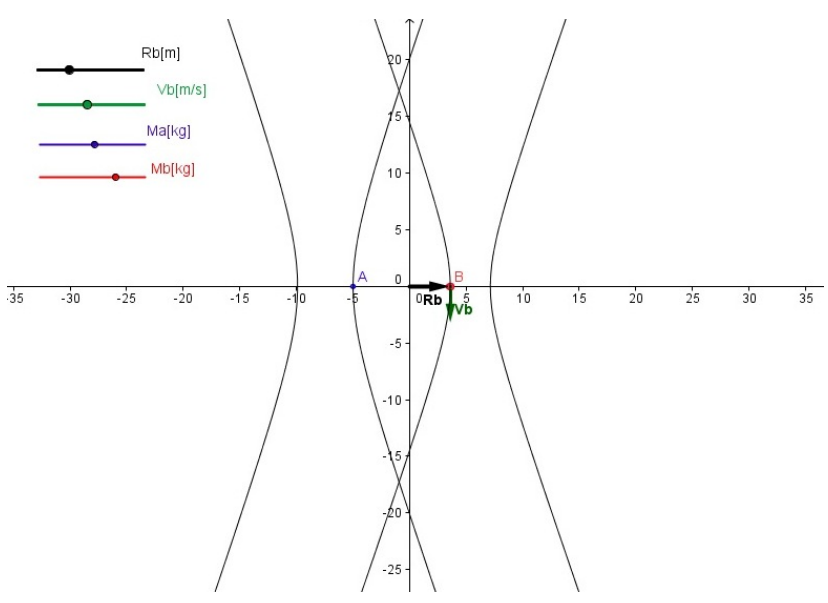

Fig. 5 Trajectory - hyperbola

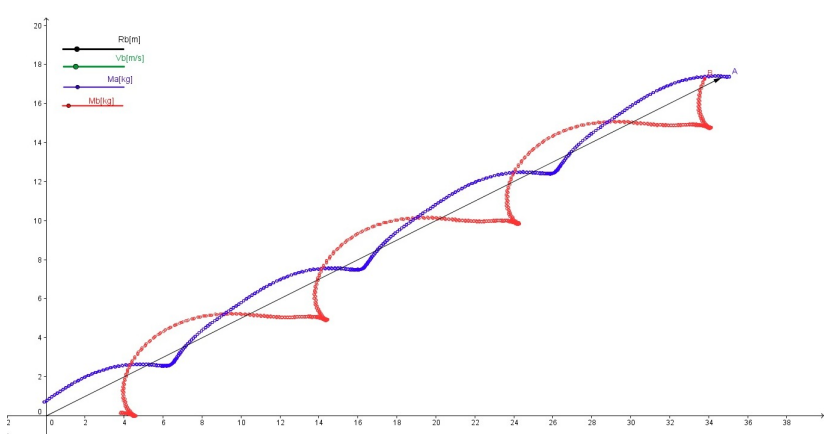

Fig. 6 Trajectory - moving center of mass

To get different kinds of trajectories, we had to change initial parameters. We used four sliders by which we changed initial values of velocity of particle $B$, it's initial position and also masses of both of the particles: Initial velocity $-V_{b}$ is varied between 0 and $7 \mathrm{~m} / \mathrm{s}$, initial position $R_{b}$ between 0 and $12 \mathrm{~m}$ and masses of the particles $B$ and $A-M_{b}$ and $M_{a}$, respectively, between 0 and $15 \mathrm{~kg}$. As our simulation is related to the movement of two arbitrary particles $A$ and $B$, four variable values, described with four sliders belong arbitrarily selected intervals. If interested reader want to simulate a concrete example of this motion, he can change magnitude of mentioned interval and also order of magnitude value of variables.

After setting this initial parameters, trajectories were shown by using locus tool. But, we also have shown how particle $A$ and $B$ move along their trajectories - you have to put Trace "on" on the both of the particles.

Since our intention was to simulate the motion of the binary system, i.e., to show its time evolution, we needed explicit expression for the angular variable $\theta$ in terms of time $t$. As shown in [2,3], calculation of the closed form of $\theta(t)$ requires thorough numerical study, and makes the problem much more involved. To avoid heavy computations we adopted another strategy. We had chosen the form of $\theta(t)$ such that the second Kepler's law - angular momentum conservation law - is approximately satisfied. It can be shown that appropriate form of the function $\theta(t)$ is:

$\theta(t)=\frac{1}{b \arctan \frac{t-a}{b}}$,

where we have taken the following values of the parameters: $a=1.23, b=0.4$. Once the explicit form of $\theta(t)$ is chosen, we can determine the change in time of polar coordinates $r_{a}(t)$ and $r_{b}(t)$, by inserting $\theta(t)$ into equations 5 . and (4), respectively.

The results of the simulations are shown in Figures $2+5$ It is obvious that the trajectories of the particles are conic sections.

It is, however, interesting to observe trajectories in another inertial frame of reference. To be precise, we can simulate the motion with respect to the system whose origin does not coincide with the center of mass (see Figure 1). In that case, we must take into account the motion of the center of mass with respect to this frame, $\vec{R}(t)=(X(t), Y(t))$, and to incorporate it into equations of motion of the particles. Particles $A$ and $B$ have following coordinates:

$A=\left(X(t)+r_{a}(t), Y(t)+\theta(t)+\pi\right) ;$

$B=\left(X(t)+r_{b}(t), Y(t)+\theta(t)\right)$

The functions $X(t)$ and $Y(t)$ must describe uniform rectilinear motion of the center of mass. The result of this simulation is given in Figure 6 and shows that particle trajectories cannot be recognized as conic sections at first sight.

\section{RESULTS/CONCLUSIONS}

We have seen that particles move along the conics and we have shown it in GeoGebra. We said at the beginning that the center of mass moves uniformly. If we observe the motion of the center of mass, we get some interesting trajectories, different from the conics (but particle still moves along the conics with respect to the center of mass), see Fig. 6

\section{ACKNOWLEDGEMENT}

This paper was supported by CEEPUS Summer School (CIII-HU-0028-05-1112 - Active Methods in Teaching and Learning Mathematics and Informatics) organized by the 
Department of Mathematics and Theoretical Informatics, Faculty of Electrical Engineering, Technical University in Košice, Slovakia.

\section{REFERENCES}

[1] MURILLO, V. C.: Ellipses $i$ forces proporcionals a l'invers del quadrat de la distáncia, Ciéncies, Vol. 8, 2007, 25-27.

[2] KOVÁCS, Z.: Modelling with Difference Equations Supported by GeoGebra: Exploring the Kepler Problem, The International Journal for Technology in Mathematics Education, Vol. 17, No. 3, 2010, 141-146.

[3] HAŠEK, R.: Numerical analysis of a planar motion: GeoGebra as a tool of investigation, North American GeoGebra Journal, Vol. 1, No. 1, 2012, 33-36.

[4] LUNN, M.: A First Course in Mechanics, Oxford University Press, New York, 1991.

Received July 27, 2012, accepted September 28, 2012

\section{BIOGRAPHIES}

Danka Lučić was born on 18. 5. 1990. She is a student of Applied mathematics at the Department of Mathematics and Informatics of the Faculty of Science at the University of Novi Sad. This year she is going to enroll masters. Conferences: GeoGebra Conference 2011 (Novi Sad, Serbia) - Graphic display of trigonometric functions; ICPS 2011 (Budapest, Hungary) - Physics and basketball; CADGME (Novi Sad, Serbia) - Simulation of two-body problem in GeoGebra; ICPS 2012 (Utrecht, The Netherlands) - Kirchhoff's analogy: gyroscopes and elastic rods.

Mario Varga was born on 6. 1. 1990. He is a student of Applied mathematics at the Department of Mathematics and Informatics of the Faculty of Science at the University of Novi Sad. This year he is going to enroll masters. Conferences: GeoGebra Conference 2011 (Novi Sad, Serbia) - Extremes and applications; ICPS 2011 (Budapest, Hungary) - Physics and basketball; CADGME (Novi Sad, Serbia) - Simulation of two-body problem in GeoGebra; ICPS 2012 (Utrecht, The Netherlands) - Kirchhoff's analogy: gyroscopes and elastic rods. 